favorable cytogenetic group had the expected longer survival when given chemotherapy + GO rather than chemotherapy alone. The Southwest Oncology Group has also failed to notice an effect of CD33 SNPs on outcome in adults (M Othus, 2018, personal communication).

Even the minority of patients who benefit from GO might benefit more from development of improved antiCD33 therapeutics. ${ }^{17}$ One possibility here is use of bispecific antibodies (BiAbs) that engage CD33 but also direct $\mathrm{T}$ cells toward AML cells. An obvious model for this approach is blinatumomab, ${ }^{18}$ a CD3/CD19 molecule built in the Bispecific T-cell Engager (BiTE) format. A first series of CD33/CD3 BiAbs, including the BiTE AMG 330 and the tandem diabody AMV-564, have recently entered clinical tests.

Like GO, all CD33 BiAbs (and other CD33-directed therapeutics) currently under investigation recognize the $\mathrm{V}$ set domain, which is located distally on CD33. However, preliminary studies with artificial CD33 molecules show that membrane proximal binding enhances the immune effector cell functions of CD33 antibodies $(R$ Walter, 2018, personal communication). Development of antibodies recognizing such proximal sites is likely to be an area of examination in GO's second and, hopefully, subsequent acts.

\section{References}

1. Walter R, Appelbaum F, Estey E, and Bernstein I Acute myeloid leukemia stem cells and CD33-targeted immunotherapy. Blood. 2012;119(26):6198-6208.

2. McKoy J, Angelotta C, Bennett C, et al. Gemtuzumab ozogamicin-associated sinusoidal obstructive syndrome (SOS): an overview from the research on adverse drug events and reports (RADAR) project. Leuk Res. 2007;31(5):599-604

3. Castaigne S, Pautas C, Terré C, et al. Effect of gemtuzumab ozogamicin on survival of adult patients with de-novo acute myeloid leukaemia (ALFA-0701): a randomised, open-label, phase 3 study. Lancet. 2012;379(9825):1508-1516.

4. de Lima M, Strom S, Keating M, et al. Implications of potential cure in acute myelogenous leukemia: development of subsequent cancer and return to work. Blood. 1997;90(12):4719-4724.
5. Lambert J, Pautas C, Terré C et al. Gemtuzumab ozogamicin for de novo acute myeloid leukemia: final efficacy and safety updates from the open-label, phase III ALFA-0701 trial. Haematologica. 2019;104(1):113-119.

6. Niktoreh N, Lerius B, Zimmermann M et al. Gemtuzumab ozogamicin in children with relapsed or refractory acute myeloid leukemia: a report by Berlin-Frankfurt-Münster study group. Haematologica. 2019;104(1):120-127.

7. Gamis AS, Alonzo TA, Meshinchi S, et al. Gemtuzumab ozogamicin in children and adolescents with de novo acute myeloid leukemia improves event-free survival by reducing relapse risk: results from the randomized phase III Children's Oncology Group trial AAML0531.J Clin Oncol. 2014;32(27):3021-3032.

8. Burnett AK, Goldstone A, Hills RK, et al. Curability of patients with acute myeloid leukemia who did not undergo transplantation in first remission. J Clin Oncol. 2013;31(10):1293-1301.

9. Estey E, Othus M, Lee SJ, Appelbaum FR, Gale RP. New drug approvals in acute myeloid leukemia: what's the best endpoint? Leukemia. 2016;30(3):521-525

10. Hills RK, Castaigne S, Appelbaum FR, et al. Addition of gemtuzumab ozogamicin to induction chemotherapy in adult patients with acute myeloid leukaemia: a meta-analysis of individual patient data from randomised controlled trials. Lancet Oncol. 2014;15(9)986-996.

11. Pollard JA, Loken M, Gerbing RB, et al. CD33 Expression and Its Association With Gemtuzumab Ozogamicin Response: Results From the Randomized Phase III Children's Oncology Group Trial AAML0531. J Clin Oncol. 2016;34(7):747-755.

12. Olombel G, Guerin E, Guy J, et al. The level of blast CD33 expression positively impacts the effect of gemtuzumab ozogamicin in patients with acute myeloid leukemia Blood. 2016;127(17) 2157-2160.

13. Khan N, Hills RK, Virgo P, et al. Expression of CD33 is a predictive factor for effect of gemtuzumab ozogamicin at different doses in adult acute myeloid leukaemia. Leukemia. 2017;31(5):1059-1068.

14. Tsimberidou A, Estey E, Cortes J, et al. Gemtuzumab, fludarabine, cytarabine, and cyclosporine in patients with newly diagnosed acute myelogenous leukemia or high-risk myelodysplastic syndromes. Cancer. 2003;97(6):1481-1487.

15. Lamba JK, Chauhan L, Shin M, et al. CD33 Splicing Polymorphism Determines Gemtuzumab Ozogamicin Response in De Novo Acute Myeloid Leukemia: Report From Randomized Phase III Children's Oncology Group Trial AAML0531. J Clin Oncol. 2017;35(23):26742682.

16. Gale R, Pope T, Wright M, et al. No evidence that CD33 splicing SNP impacts the response to GO in younger adults with AML treated on UK MRC/NCRI trials. Blood. 2018;131(4):468-471.

17. Walter $R$ Investigational CD33-targeted therapeutics for acute myeloid leukemia. Expert Opin Investig Drugs 2018; 27(4): 339-348

18. Kantariian H, Stein A, Gökbuget N, et al. Blinatumomab versus Chemotherapy for Advanced Acute Lymphoblastic Leukemia. N Engl J Med. 2017;376(9):836-847.

\title{
Lenalidomide can be safely combined with chlorambucil and rituximab in older patients with chronic lymphocytic leukemia
}

\section{Candida Vitale ${ }^{1}$ and Alessandra Ferrajoli ${ }^{2}$}

'Division of Hematology, University of Torino, A.O.U. Città della Salute e della Scienza di Torino, Italy and 'Department of Leukemia, The University of Texas MD Anderson Cancer Center, Houston, TX, USA

\section{E-mail:aferrajo@mdanderson.org}

doi:10.3324/haematol.2018.206359

T the clinical activity of lenalidomide in chronic lymphocytic leukemia (CLL) was first reported more than 10 years ago. ${ }^{1,2}$ Since then, this agent has been studied in various combinations with anti-CD20 monoclonal antibodies, chemotherapy, chemo-immunotherapy and B-cell receptor (BCR)-targeting agents. These studies have shown clinical responses; however, most importantly, they have also highlighted unique and unexpected toxicities, in particular when lenalidomide was combined with chemo-immunotherapy and targeted agents.

In this issue of Haematologica, Kater and colleagues report the experience of the HOVON CLL study group on the feasibility and efficacy of the combination of lenalidomide, chlorambucil, and rituximab in treatment-naïve patients 
with CLL. ${ }^{3}$ The patients enrolled in this trial were considered ineligible to receive the combination of fludarabine, cyclophosphamide, and rituximab (FCR) because of their older age or the presence of comorbidities. For the first six cycles (induction-I), lenalidomide was given in combination with chlorambucil and rituximab at a starting dose of 2.5 $\mathrm{mg}$, with escalation to $10 \mathrm{mg}$. The authors report that they were able to administer a median lenalidomide dose of $86.7 \%$ of the full dose, with the full dose given to more than $50 \%$ of patients. For the next six cycles (induction-II), lenalidomide was given as monotherapy at a dose of $10 \mathrm{mg}$ daily. The median administered dose was $99.7 \%$ of the full dose, and the full dose was given to $69 \%$ of patients during cycle 6 . The results of this phase 1-2 study showed that the combination of lenolidamide, chlorambucil, and rituximab can be safely administered to patients with CLL: grade 3-4 toxicities were mainly hematologic (grade 3-4 neutropenia occurred in $73 \%$ and $64 \%$ of patients during induction-I and induction-II, respectively), tumor lysis syndrome did not occur, tumor flare reaction occurred in five (9\%) patients (mainly grade 2), and two (4\%) patients had a thromboembolic event despite thromboembolic prophylaxis. Of 53 patients in induction-I, eight discontinued treatment because of excessive toxicity, whereas five of 42 patients discontinued treatment during induction-II. The authors also report on the activity of this combination:

A

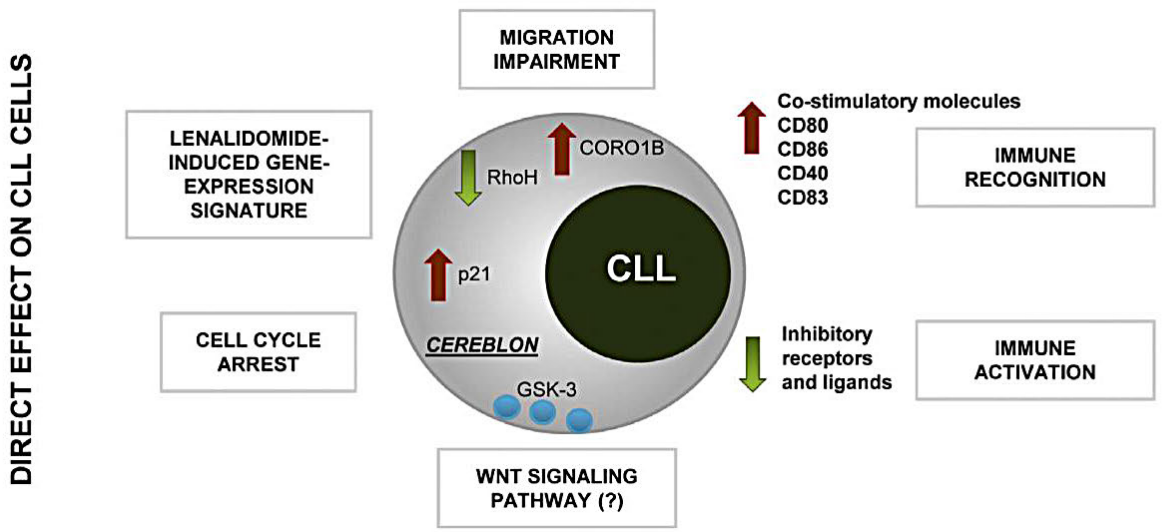

B

$\begin{array}{ll}\text { M2 to M1 switch } & \text { CD163 } \\ \text { Phagocytosis } & \text { IGF1 } \\ \text { Induction of T cell proliferation } & \checkmark \text { CXCL12 } \\ \text { Antigen presentation } & \text { CCL2 }\end{array}$
Antigen presentation

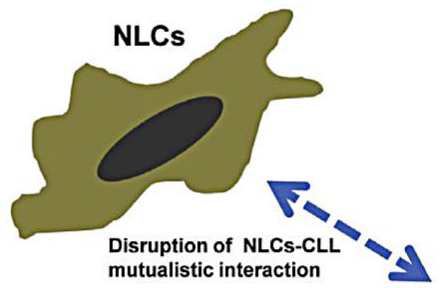

Th2 to Th1 switch
Repair of immune synapse
impairment

Increase of cytotoxic potential

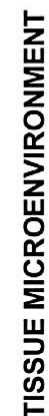
mutualistic interaction

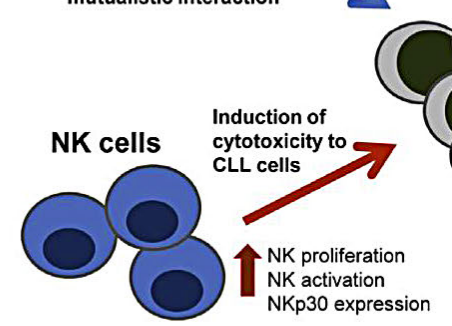

CLL

Mobility and adhesion

C

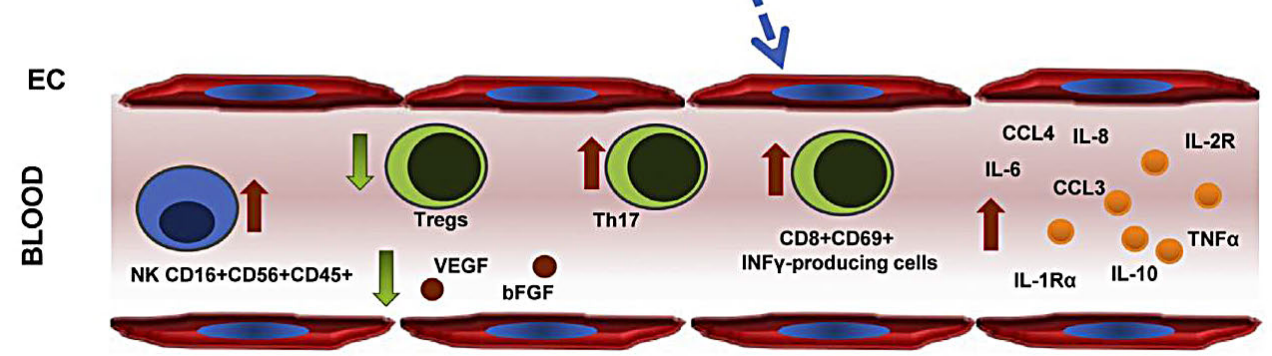

Figure 1. Mechanisms of action of lenalidomide. The mechanisms of action of lenalidomide include: (A) direct effects on CLL cells and $(B, C)$ modification of tumor-microenvironment interactions. This figure is reproduced with permission from Mattei $\mathrm{R}$ et al., Lenalidomide in chronic lymphocytic leukemia: the present and future in the era of tyrosine kinase inhibitors. Crit Rev Oncol Hematol. 206;97:291-302. NLCs: nurse-like cells; $\mathrm{EC}$ : endothelial cells. 
responses were seen in $83 \%$ of the patients treated, the median progression-free survival was 49 months, and the 3 year overall survival rate was $95 \%$.

Early monotherapy trials showed that lenalidomide is associated with a unique toxicity profile in patients with CLL, causing tumor lysis syndrome, tumor flare reaction, myelosuppression, and, in particular, neutropenia, skin rash, and diarrhea. ${ }^{1,2}$ Particularly severe events, including deaths, were reported in trials with no dose escalation ${ }^{4}$ or rapid dose escalation, ${ }^{5}$ and tumor lysis syndrome occurred in patients with bulky lymphadenopathy despite proper prophylaxis. ${ }^{1}$ After these early experiences, trials with lenalidomide employed stepwise dose escalation strategies with low starting doses (usually $2.5 \mathrm{mg} /$ day), as in the study presented by Kater et al., and managed tumor flare reactions with non-steroidal anti-inflammatory drugs and corticosteroids. Moreover, careful patient selection is recommended. The population included in the study by Kater et al. mainly consisted of older but fit patients: 98\% were 65 years or older, $87 \%$ had a Cumulative Illness Rating Scale score of 6 or lower, and all had a glomerular filtration rate of $60 \mathrm{~mL} / \mathrm{min}$ or higher at the time of entry into the study.

In the last decade, several lenalidomide-containing combination regimens have been evaluated in patients with treatment-naïve CLL. When lenalidomide was combined with rituximab in the frontline setting, the treatment was generally well tolerated, with the most common grade 3-4 toxicities being neutropenia, anemia, infections, increased transaminase levels, and skin rash. ${ }^{6,7}$ However, when different partners, such as chemotherapy agents or targeted drugs, were tested in combination with lenalidomide, some trials documented excessive toxicities that led to early termination of the studies. For instance, a phase 1 study investigating the combination of lenalidomide with fludarabine and rituximab was closed early because of unpredictable reactions and unexpectedly persistent myelosuppression, even when very low doses of fludarabine and lenalidomide were given, which made treatment delivery difficult. ${ }^{8}$ Instead, induction treatment with low-dose lenalidomide together with reduced-dose FCR was demonstrated to be safe. ${ }^{9}$ In the relapse setting, lenalidomide was evaluated in association with rituximab and ibrutinib; the study investigating this approach showed a high incidence of persistent severe neutropenia that occurred despite growth factor support. ${ }^{10}$ This unfavorable toxicity profile, together with poor preliminary efficacy data, discouraged further evaluation of this combination. The combination of lenalidomide with rituximab and idelalisib also showed unacceptable liver toxicity in patients with relapsed or refractory indolent lymphoma. ${ }^{11}$

Regarding efficacy, the single-arm design of the study by Kater et al. does not allow a direct comparison of the triple combination with chlorambucil and rituximab. Acknowledging the limitations of cross-trial comparisons, however, the efficacy of the proposed regimen compares positively with that of chlorambucil plus anti-CD20 monoclonal antibodies. In a study conducted by Strati et al., the combination of lenalidomide plus rituximab produced an overall response rate of $73 \%$ in treatment-naive patients, with a complete remission (CR)/CR with incomplete hematologic recovery (CRi) rate of $35 \%$, a median time to treatment failure of 22 months, and a 4-year overall survival rate of $90 \%$. The same treatment combination was explored in a multicenter study, which showed an overall response rate of $88 \%$, of which $15 \%$ were CR/CRi, and a median progression-free survival of 19 months. ${ }^{6}$

It is essential to put the data presented by Kater and colleagues into perspective by considering recent changes in the treatment landscape of CLL brought about by the availability of new targeted drugs, such as BTK inhibitors, PI3K inhibitors, and Bcl-2 antagonists, which have also been studied in older patients. In a recent update of the phase III RESONATE-2 trial of ibrutinib, which enrolled patients aged 65 years and older with previously untreated CLL and without del(17p), researchers reported that at a median follow-up time of 29 months, the overall response rate was $92 \%$, the median duration of progression-free survival had not been reached, and the 24-month progression-free survival rate was $89 \% .{ }^{12}$ Patients carrying abnormalities on chromosome 17 represent a subset of CLL patients with a particularly poor prognosis. In the cohort presented by Kater et al., eight (17\%) patients had $\operatorname{del}(17 \mathrm{p})$, and their progression-free survival rate was lower than that of patients without del(17p) (38\% versus $59 \%$ at 3 years). Notably, in a phase II study that evaluated ibrutinib in a cohort of treatment-naive CLL patients with TP53 aberrations, the estimated 5-year progression-free survival rate was $74.4 \% .{ }^{13}$

That being said, a credit that pertains exclusively to lenalidomide is the role this drug has had in elucidating tumor-microenvironment interactions in CLL. Phenotypic and functional immune defects are known to be associated with CLL; these defects confer an increased risk of infections and autoimmune phenomena and foster leukemia cell proliferation and survival. Several studies have shown that treatment with lenalidomide modulates the cross-talk between tumor cells and various components of the tumor microenvironment. Examples of these effects include the ability to normalize $\mathrm{CD}^{+} \mathrm{T}$-cell and $\mathrm{T}_{\text {reg }}$ numbers in vivo ${ }^{14,15}$ and to restore immunological synapse formation. ${ }^{16}$ The antitumoral activities of lenalidomide also appear to be attributable to a direct effect on neoplastic cells; lenalidomide not only enhances immune recognition, but also induces CRBN-mediated upregulation of p21 in vitro ${ }^{17}$ (Figure 1).

The recent progresses in immunotherapy approaches that exploit the ability to engineer the T-cell receptor, such as chimeric antigen receptor (CAR) T-cell therapy, may revitalize interest in the use of immunomodulatory agents, including lenalidomide, in CLL. Immune dysfunctions are thought to be responsible for the lower efficacy of these approaches in CLL than in other lymphoproliferative diseases. Apheresis products from CLL patients and the derived CAR T-cell products exhibit an exhausted phenotype and tend to have reduced potency. It has been demonstrated that certain features of the apheresis product, such as the predominance of early memory/naive $T$ cells and low expression of exhaustion markers, correlate with efficacy. ${ }^{18}$ It has also been reported that ibrutinib may correct some of the T-cell defects that hinder CAR T-cell production and enhance in vivo function. ${ }^{19}$ The ability of lenalidomide to enhance CAR T-cell activity has been explored in a mouse model of $\mathrm{B}$-cell lymphoma $\mathrm{a}^{20}$ and provides a rationale for future investigations of the immunomodulatory properties of lenalidomide and its derivatives in CLL. 


\section{References}

1. Chanan-Khan A, Miller KC, Musial L, et al. Clinical efficacy of lenalidomide in patients with relapsed or refractory chronic lymphocytic leukemia: results of a phase II study. J Clin Oncol. 2006;24(34):53435349.

2. Badoux XC, Keating MJ, Wen S, et al. Lenalidomide as initial therapy of elderly patients with chronic lymphocytic leukemia. Blood. 2011;118(13):3489-3498

3. Kater AP, van Oers MHJ, van Norden Y, et al. Feasibility and efficacy of addition of individualized-dose lenalidomide to chlorambucil and rituximab as first-line treatment in elderly and FCR-unfit patients with advanced chronic lymphocytic leukemia. Haematologica. 2019;104 (1):147-154.

4. Andritsos LA, Johnson AJ, Lozanski G, et al. Higher doses of lenalidomide are associated with unacceptable toxicity including life-threatening tumor flare in patients with chronic lymphocytic leukemia. J Clin Oncol. 2008;26(15):2519-2525

5. Chen CI, Bergsagel PL, Paul H, et al. Single-agent lenalidomide in the treatment of previously untreated chronic lymphocytic leukemia. J Clin Oncol. 2011;29(9):1175-1181.

6. James DF, Werner L, Brown JR, et al. Lenalidomide and rituximab for the initial treatment of patients with chronic lymphocytic leukemia: a multicenter clinical-translational study from the Chronic Lymphocytic Leukemia Research Consortium. J Clin Oncol. 2014;32(19):2067-2073.

7. Strati P, Thompson PA, Keating M, et al. A Phase II Study of the combination of lenalidomide and rituximab in patients with treatmentnaiVe and relapsed chronic lymphocytic leukemia. Blood. 2016;128(22):4389-4389.

8. Brown JR, Abramson J, Hochberg E, et al. A phase I study of lenalidomide in combination with fludarabine and rituximab in previously untreated CLL/SLL. Leukemia. 2010;24(11):1972-1975.

9. Mato AR, Foon KA, Feldman $\mathrm{T}$, et al. Reduced-dose fludarabine, cyclophosphamide, and rituximab (FCR-Lite) plus lenalidomide, followed by lenalidomide consolidation/maintenance, in previously untreated chronic lymphocytic leukemia. Am J Hematol. 2015;90 (6):487-492.
10. Ujjani C, Wang H, Skarbnik A, et al. A phase 1 study of lenalidomide and ibrutinib in combination with rituximab in relapsed and refractory CLL. Blood Adv. 2018;2(7):762-768.

11. Cheah CY, Nastoupil LJ, Neelapu SS, Forbes SG, Oki Y, Fowler NH Lenalidomide, idelalisib, and rituximab are unacceptably toxic in patients with relapsed/refractory indolent lymphoma. Blood. 2015;125(21):3357-3359.

12. Barr PM, Robak T, Owen C, et al. Sustained efficacy and detailed clinical follow-up of first-line ibrutinib treatment in older patients with chronic lymphocytic leukemia: extended phase 3 results from RESONATE-2. Haematologica. 2018;103(9):1502-1510.

13. Ahn IE, Farooqui MZH, Tian X, et al. Depth and durability of response to ibrutinib in CLL: 5-year follow-up of a phase 2 study. Blood. 2018;131(21):2357-2366.

14. Lee B-N, Gao H, Cohen EN, et al. Treatment with lenalidomide modulates T-cell immunophenotype and cytokine production in patients with chronic lymphocytic leukemia. Cancer. 2011;117(17):3999-4008.

15. Strati P, Keating MJ, Wierda WG, et al. Lenalidomide induces long-lasting responses in elderly patients with chronic lymphocytic leukemia. Blood. 2013;122(5):734-737.

16. Ramsay AG, Johnson AJ, Lee AM, et al. Chronic lymphocytic leukemia T cells show impaired immunological synapse formation that can be reversed with an immunomodulating drug. J Clin Invest. 2008;118(7):2427-2437.

17. Fecteau JF, Corral LG, Ghia EM, et al. Lenalidomide inhibits the proliferation of CLL cells via a cereblon/p21(WAF1/Cip1)-dependent mechanism independent of functional p53. Blood. 2014;124(10):1637-1644.

18. Fraietta JA, Lacey SF, Orlando EJ, et al. Determinants of response and resistance to CD19 chimeric antigen receptor (CAR) T cell therapy of chronic lymphocytic leukemia. Nat Med. 2018;24(5):563-571.

19. Fraietta JA, Beckwith KA, Patel PR, et al. Ibrutinib enhances chimeric antigen receptor T-cell engraftment and efficacy in leukemia. Blood. 2016;127(9):1117-1127.

20. Otahal P, Prukova D, Kral V, et al. Lenalidomide enhances antitumor functions of chimeric antigen receptor modified $\mathrm{T}$ cells Oncoimmunology. 2016;5(4):e1115940. 